\title{
Las competencias profesionales adquiridas por los alumnos durante la Formación Inicial del Profesorado: Cambios en la percepción del alumnado
}

Carmen Merino Merino. Universidad de Valladolid

Inés Monreal Guerrero. Universidad de Valladolid

María de la O Cortón de las Heras. Universidad de Valladolid

Recepción: 17.07.2019 | Aceptado: 15.10.2019

Correspondencia a través de ORCID: Carmen Merino

0000-0002-4231-6906

Citar: Merino, C., Monreal, I. y Cortón, María de la O. (2019). Las competencias profesionales adquiridas por los alumnos durante la Formación Inicial del Profesorado: Cambios en la percepción del alumnado. REIDOCREA, 8, 212-220.

Resumen: Los cambios requeridos por el Plan Bolonia, tanto en los planes de estudio como en la metodología a la hora de formar al alumnado, han llevado al profesor universitario a considerar el trabajo por competencias. En este estudio, partimos de la definición y tipología de competencias docentes propuestas por Perrenoud para analizar, mediante métodos mixtos que incorporan técnicas de investigación como el cuestionario y las entrevistas semiestructuradas, la percepción de la adquisición de competencias profesionales durante la formación inicial del profesorado entre los alumnos del plan antiguo de la Diplomatura de Magisterio y las dos primeras promociones del plan nuevo del Grado de Educación en el campus de Segovia, perteneciente a la Universidad de Valladolid. Los resultados obtenidos revelan un cambio en la percepción de la adquisición de competencias por parte del alumnado a lo largo de los tres momentos de realización del trabajo de campo, aunque se mantiene la tendencia a formar a los futuros docentes en aquellas competencias más propias del trabajo dentro del aula, en detrimento de aquellas otras que implicarían mayor relación con el entorno y con otros miembros de la comunidad educativa.

Palabras clave: Competencias del docente | Formación de docentes

The Professional Competences acquired by Students during the Initial Teacher Training: shifts in students' perception

\begin{abstract}
The changes required by the Bologna Plan, both in the curricula and in the methodology when training students, have led professors to consider approaches by competencies. In this study, we will start from the definition and typology of teaching competences proposed by Perrenoud to analyze, through mixed methods that incorporate research techniques such as the questionnaire and half-structured interviews, the perception of the acquisition of professional competences during the Initial Teacher Training in the students belonging to both the old plan in Education and the first two promotions of the new Degree in Education at Segovia Campus, University of Valladolid. Therefore, the obtained results reveal a shift in the perception of the acquisition of competences by students during the three moments of the fieldwork. However, the tendency to train future teachers in those competences which are more typical of the work in the classroom is maintained at the expense of those that would imply a greater relationship with the environment and with other members of the educational community.
\end{abstract}

Keywords: Teacher qualifications | Teacher training

\section{Introducción}

Hoy en día "la formación inicial y continua de los docentes, desde la etapa de la educación infantil hasta la de la educación secundaria postobligatoria, es hoy un tema de especial preocupación en muchos países y de especial reflexión por parte de muchas universidades y administraciones educativas" (Martínez, 2016) puesto que los estudios sobre la formación del profesorado pueden contribuir de forma eficiente a eliminar los desajustes entre el diseño inicial de dicha formación y las demandas de la sociedad.

El Plan Bolonia ha contribuido a la transformación de la formación inicial del profesorado hacia una educación más centrada en el aprendizaje del estudiante y en las competencias docentes que debe adquirir para el ejercicio de su profesión. En este sentido, y siguiendo a Pimienta Prieto (2012) cabe decir que "es posible afirmar que, en la actualidad, la labor del profesor debe dirigirse al diseño de tareas docentes que 
provoquen la actividad de los estudiantes, con la finalidad de contribuir a la formación de las competencias deseables."

Esto supone nuevos retos para los docentes que deben basar este cambio en la formación por competencias, insertos en un debate a favor y en contra de las propias competencias. Zabalza (2012) identifica tres tipos de discursos que se han ido elaborando y solapando entorno a ellas: el discurso conceptual, el técnico y el cultural. Siguiendo al mismo autor, el discurso conceptual se ha centrado en valorar la "legitimidad de este enfoque" con debates como la influencia de los planteamientos neoliberales y la supeditación de las universidades a los mercados o si la apuesta por este enfoque implica o no una fuerte pérdida de conocimientos para el alumnado. Por otro lado, el discurso técnico que identifica Zabalza se afana en "planificar su desarrollo y establecer los dispositivos necesarios para incorporarlas al diseño curricular, a la organización de los procesos formativos, a los sistemas de evaluación”. Mientras que el discurso cultural, el más relevante para el autor (siguiendo a Darling-Hammond, 2009), "establece el marco actitudinal y cognitivo sin el cual cualquiera de los otros dos resulta inoperante" al centrarse en la actitud positiva (o no) al cambio que trabajar por competencias implica en el quehacer de profesores y estudiantes.

De entre todos los discursos y clasificaciones, nos hemos basado para este estudio en la definición e identificación de competencias docentes que las aportaciones del sociólogo Philippe Perrenoud realiza. Este autor define la competencia docente como el "conjunto de conocimientos, actitudes, destrezas, sentimientos, habilidades (denominados recursos cognitivos) movilizados por el profesor para responder a demandas complejas en situaciones específicas del proceso de enseñanza-aprendizaje, apoyándose en los recursos externos que se tengan a su alcance" (Perrenoud, 1999). Más tarde, el mismo autor concebirá las competencias como "una capacidad de movilizar varios recursos cognitivos para hacer frente a un tipo de situaciones" (Perrenoud, 2004) poniendo de relieve los elementos implícitos que coexisten en la propia definición como la existencia de diferentes tipos de situaciones, los propios recursos movilizados, los esquemas de pensamiento e incluso la aplicación cotidiana de cada individuo en el trabajo, entre otros.

En esta misma obra, Perrenoud (2004) elabora una propuesta de tipología de competencias, realizando una agrupación de competencias en diez grupos de familias, cada una de ellas estrechamente ligada a determinados tipos de problemas y tareas más específicas. Esta agrupación la realiza en base al referencial adoptado en Ginebra en 1996 y ha sido ampliada en nuestra investigación con otras tres más, al otorgarles gran importancia respecto a la formación de nuestros futuros docentes (ver Tabla 1).

En este estudio pretendemos abordar de forma muy general, la percepción que tienen distintas promociones de alumnos sobre la adquisición de las competencias seleccionadas, en unos momentos en los que tanto el trabajo como el perfil del profesional docente se ve abocado a una profunda redefinición, emergiendo "la necesidad de la práctica reflexiva en el oficio de enseñar" que implicaría también "el buen conocimiento de los contenidos a enseñar y de su didáctica, pero también conocimiento de la profesión y de sí mismo" (Pavié, 2011).

Tabla 1. Identificación de las competencias docentes. 


\begin{tabular}{|c|c|c|}
\hline \multicolumn{2}{|c|}{ Id } & Competencias \\
\hline \multirow{10}{*}{ 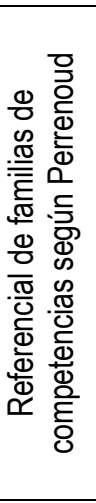 } & C1 & Organizar y animar situaciones de aprendizaje \\
\hline & $\mathrm{C} 2$ & Gestionar la progresión de los aprendizajes \\
\hline & C3 & Elaborar y poner en práctica estrategias de atención a la diversidad \\
\hline & $\mathrm{C} 4$ & Implicar al alumnado en su aprendizaje y en la vida del centro \\
\hline & $\mathrm{C5}$ & Trabajar en equipo con otros docentes \\
\hline & C6 & Participar en la gestión del Centro \\
\hline & $\mathrm{C7}$ & Informar e implicar a las familias \\
\hline & C8 & Utilizar las tecnologías de la información y la comunicación \\
\hline & $\mathrm{Cg}$ & Afrontar los deberes y los dilemas éticos de la profesión \\
\hline & C10 & Organizar la propia formación continua \\
\hline \multirow{3}{*}{ 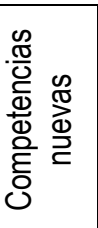 } & C11 & Fomentar el interés por la lectura \\
\hline & $\mathrm{C} 12$ & Fomentar la educación en valores \\
\hline & C13 & Elaborar diseños curriculares (programaciones y unidades didácticas.) \\
\hline
\end{tabular}

\section{Objetivos o hipótesis}

Dos objetivos principales: Analizar, mediante cuestionario, las diferencias en la percepción de la adquisición de competencias profesionales durante su formación inicial, por parte de los alumnos de la última promoción del antiguo plan de Estudios de Magisterio en relación con los alumnos de la primera promoción de Grado de Educación Infantil y Primaria de la actual Facultad de Educación de Segovia.

Analizar cómo el ejercicio de la profesión entre los alumnos de la primera y segunda promoción de Grado ha influido en la percepción de adquisición de competencias profesionales, mediante el diseño y análisis de entrevistas personales semiestructuradas.

Las hipótesis del estudio son dos:

Hipótesis 1: Existen diferencias significativas en la percepción de la adquisición de competencias entre los alumnos del plan de estudios antiguo y los del plan nuevo.

Hipótesis 2: El ejercicio profesional ha variado la percepción de la adquisición de competencias entre los alumnos de las dos primeras promociones del Grado de Educación Infantil y Primaria.

\section{Métodos}

Para el diseño metodológico se ha elaborado a partir de la combinación de métodos cuantitativos y cualitativos de investigación, diseñando una metodología mixta entre el paradigma positivista y el interpretativo. Las técnicas utilizadas para conseguir los objetivos propuestos son: la encuesta y la entrevista telefónica semiestructurada, respectivamente.

\section{Participantes}


El universo poblacional del estudio cuantitativo son los alumnos y alumnas del campus universitario de Segovia, perteneciente a la Universidad de Valladolid, del último curso de titulación de Diplomatura de Magisterio, correspondiente al plan antiguo (curso académico 2008-09) y de la titulación de Grado en Educación como del plan nuevo (cursos académicos 2012-14), con edades comprendidas entre los 21 y los 49 años.

Tabla 1. Distribución del tamaño muestral según Centro y Curso académico.

\begin{tabular}{|c|c|c|c|}
\hline Universidad & $\begin{array}{c}\text { Curso } \\
\text { académico }\end{array}$ & Titulación y Centro & $\begin{array}{l}\text { Tamaño } \\
\text { muestral }\end{array}$ \\
\hline \multirow{3}{*}{ 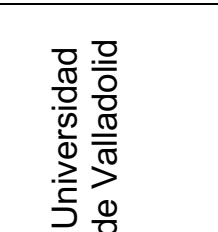 } & $2008-09$ & $\begin{array}{l}\text { Diplomatura en Magisterio } \\
\text { E.U. de Magisterio del Campus de Segovia }\end{array}$ & 205 \\
\hline & $2012-13$ & \multirow{2}{*}{$\begin{array}{l}\text { Grado en Educación } \\
\text { Facultad de Educación del Campus de } \\
\text { Segovia }\end{array}$} & 136 \\
\hline & $2013-14$ & & 1116 \\
\hline
\end{tabular}

En la selección del alumnado se ha tenido en cuenta a todo el universo poblacional objeto de estudio. Se ha creído oportuno administrar el cuestionario a estudiantes del último curso durante el mes de mayo (y en relación con los alumnos de Grado durante las dos primeras promociones) para poder obtener información acerca de su formación inicial al finalizar todo el periodo formativo, en las distintas titulaciones y especialidades/menciones.

Los participantes en el estudio cualitativo son los egresados de las dos primeras promociones del Plan Nuevo del Grado en Educación, que ejercen o han ejercido la profesión hasta el mes de mayo de 2019. La selección de unidades finales de la muestra estructural se realizó en base a las siguientes variables: años de experiencia profesional, tipo de colegio en el que se ha trabajado (público, privado o concertado). En total se han realizado, hasta conseguir la saturación discursiva, 16 entrevistas semiestructuradas.

Tabla 2. Distribución de entrevistas según los ejes años de experiencia y tipo de colegio.

\begin{tabular}{|c|l||l|l|}
\hline Años de experiencia & Hasta 3 años & 3 y más años \\
\hline \hline \multirow{2}{*}{$\begin{array}{l}\text { Tipo de } \\
\text { colegio }\end{array}$} & Público & 5 & 6 \\
\cline { 2 - 4 } & Privado/Concertado & 3 & 2 \\
\hline
\end{tabular}

\section{Instrumentos}

Para la realización de la investigación se han utilizado dos instrumentos: el cuestionario autocumplimentado y la entrevista telefónica semiestructurada.

El cuestionario se estructuró en varios apartados: Uno de ellos recoge las variables de cabecera (sexo, edad, vía de acceso a la titulación, especialidad/mención, etc) y otro bloque con la batería de preguntas sobre la percepción de las competencias docentes, la utilización de la evaluación formativa y el empleo de metodologías activas durante la formación inicial del profesorado. Para este último bloque se utilizó la variable escala como instrumento de medición.

En el diseño de las entrevistas semiestructuradas se mantuvo el mismo criterio respecto a los bloques de contenidos para facilitar la comparabilidad de la información recogida, 
a excepción del bloque con las preguntas de cabecera que incluía variables como: años de experiencia, relación contractual, tipo de centro en el que trabaja (público, privado o concertado), etapa, nivel y/o curso en los que ha trabajado, experiencia en tutoría y comunidad autónoma donde ejerce o ha ejercido la profesión).

\section{Procedimiento}

Para garantizar el rigor científico en el diseño del cuestionario se llevaron a cabo procesos de revisión por expertos y por el alumnado en la fase de elaboración del pretest, con el fin de garantizar la comprensión de la batería de preguntas y la inclusión de respuestas exhaustivas y mutuamente excluyentes.

El trabajo de campo de las sucesivas encuestas se llevó a cabo, como periodo de referencia, en la tercera semana del mes de mayo de 2009, 2013 y 2014. Los cuestionarios se facilitaron a los alumnos para su autocumplimentación durante el transcurso de una clase y bajo la supervisión de un investigador. Una vez finalizado el trabajo de campo se procedió a la codificación, grabación, supervisión y anonimización de la matriz de datos.

El trabajo de campo de las entrevistas cualitativas se realizó durante los meses de abrilmayo de 2019, incluyendo las fases de contactación, realización, transcripción de las entrevistas y análisis e interpretación de los datos.

La explotación de los datos cuantitativos recogidos a través de los cuestionarios ha sido realizada con el programa informático SPSS mientras que, para un análisis más profundo del análisis de los datos cualitativos, está previsto utilizar el programa Atlas.ti.

Nosotros aquí presentamos los resultados de un primer análisis general del cuestionario en lo que se refiere a las cuestiones que versan sobre la percepción de la adquisición de las competencias profesionales durante la formación inicial del profesorado en los dos grupos de población analizados: alumnado de la antigua titulación de Diplomatura de Magisterio correspondiente al plan antiguo y el alumnado de la primera y segunda promoción del Grado de Educación del plan nuevo.

\section{Análisis de datos}

En el análisis de datos cuantitativos, se parte de la hipótesis de que las medias son distintas en las variables de percepción de adquisición de Competencias docentes según el Plan de estudios que ha cursado el alumnado. Para poder rechazar la hipótesis nula, que afirma que las medias son similares en los distintos grupos, se realizan por orden las siguientes pruebas:

1- Primero se examinan las variables con sus descriptivos y diagramas de cajas.

2- Se comprueban los requisitos de normalidad y homocedasticidad.

3- Se realizan pruebas paramétricas como la prueba $T$ de Student para variables de clasificación con dos grupos o categorías.

4- $\mathrm{Y}$, por último, se realizan pruebas no paramétricas. Para ello se piden los estadísticos U de Mann-Whitney y W de Wilcoxon para variables de clasificación con dos grupos.

Los resultados de la prueba de normalidad de Kolmogorov-Smirnov nos muestran que son todos significativos, luego las variables no se distribuyen según la Ley Normal. Para el requisito de homocedasticidad se pide la prueba de Levene para la igualdad de varianzas, en la que se observa como seis de las 15 variables tampoco cumplen el requisito de homocedasticidad, por lo que se duda que tengan varianzas homogéneas. 
Al observar que las diferencias de medias son negativas en la prueba $T$ para igualdad de medias y que los Intervalos de Confianza para las diferencias de medias al $95 \%$ no contienen el valor cero podemos concluir que las diferencias son estadísticamente significativas. En cualquier caso, pasamos a realizar pruebas no paramétricas para todas las variables analizadas en el presente estudio con la prueba de $U$ de MannWhitney y la W de Wilcoxon.

En todos los casos la Significación Asintótica (bilateral) es menor que 0,05 por lo que se rechaza la hipótesis nula de que la media es similar en ambos Planes de estudio. Esto quiere decir que existe una asociación estadísticamente significativa entre el plan de estudios cursado y la percepción de la adquisición en cada una de las competencias docentes analizadas en el estudio.

Tabla 3. Estadísticos de contraste ${ }^{a}$ de las pruebas no paramétricas, para cada una de las variables de percepción de las competencias docentes.

aariable de agrupación: Plan de estudios.

\begin{tabular}{|l||c|c|c|r||}
\hline \multicolumn{1}{|c|}{$\begin{array}{c}\text { U de } \\
\text { Competencias docentes }\end{array}$} & $\begin{array}{c}\text { W W de } \\
\text { Whitney }\end{array}$ & Wilcoxon & Z & $\begin{array}{c}\text { Sig. } \\
\text { asintót. } \\
\text { (bilateral) }\end{array}$ \\
\hline \hline C1 & 13569,500 & 34479,500 & $-9,270$ &, 000 \\
\hline \hline C2 & 12538,500 & 33041,500 & $-9,785$ &, 000 \\
\hline \hline C3 & 17063,500 & 37973,500 & $-6,576$ &, 000 \\
\hline \hline C4 & 17025,500 & 37731,500 & $-6,536$ &, 000 \\
\hline C5 & 17395,500 & 38305,500 & $-6,221$ &, 000 \\
\hline \hline C6 & 17425,000 & 38335,000 & $-6,359$ &, 000 \\
\hline C7 & 18809,500 & 39719,500 & $-5,213$ &, 000 \\
\hline \hline C8 & 10506,500 & 31416,500 & $-11,299$ &, 000 \\
\hline \hline C9 & 20240,000 & 40946,000 & $-4,000$ &, 000 \\
\hline \hline C10 & 18433,500 & 39345,500 & $-5,267$ &, 000 \\
\hline \hline C11 & 20538,500 & 41244,500 & $-3,875$ &, 000 \\
\hline \hline C12 & 17696,500 & 38199,500 & $-5,935$ &, 000 \\
\hline \hline C13 & 16907,500 & 37817,500 & $-6,777$ &, 000 \\
\hline
\end{tabular}

La interpretación de los discursos de las entrevistas se realiza tras la identificación y agrupación de los fragmentos discursivos de cada una de ellas, organizados por cada uno de los bloques temáticos de contenido contemplados y la identificación de palabras clave para el estudio. Los bloques de contenidos no contemplados inicialmente y que surgieron en el transcurso de las entrevistas también han sido identificados en el análisis.

\section{Resultados}

Los alumnos del Grado de Educación del Campus de Segovia de la Universidad de Valladolid, que han estudiado con los nuevos planes de estudios tras la implantación del 
Plan Bolonia, perciben más claramente que con las asignaturas del Plan Nuevo han adquirido en mayor medida las competencias docentes analizadas en el estudio a lo largo de toda su formación inicial, a excepción de las competencias "Afrontar los deberes y los dilemas éticos de la profesión" y "Organizar la propia formación continua" (incluso a pesar de ser las dos variables con mayor dispersión entre sus datos), que son percibidas en mayor medida entre los alumnos del Plan Antiguo.

Tabla 4. Diferencia de medias para cada una de las variables de percepción de las competencias docentes.

\begin{tabular}{|c|c|c|c|c|}
\hline \multirow[b]{2}{*}{ Competencias docentes } & \multicolumn{2}{|c|}{ Media } & \multicolumn{2}{|c|}{ Desviación Típica } \\
\hline & $\begin{array}{l}\text { Plan } \\
\text { Antiguo }\end{array}$ & $\begin{array}{l}\text { Plan } \\
\text { Nuevo }\end{array}$ & $\begin{array}{l}\text { Plan } \\
\text { Antiguo }\end{array}$ & $\begin{array}{l}\text { Plan } \\
\text { Nuevo }\end{array}$ \\
\hline $\mathrm{C} 1$ & 1,55 & 2,31 & 0,749 & 0,831 \\
\hline $\mathrm{C} 2$ & 1,40 & 2,23 & 0,781 & 0,878 \\
\hline $\mathrm{C} 3$ & 1,44 & 2,08 & 0,884 & 1,001 \\
\hline $\mathrm{C4}$ & 1,43 & 2,05 & 0,901 & 0,980 \\
\hline C5 & 1,42 & 2,13 & 1,089 & 1,208 \\
\hline C6 & 0,75 & 1,29 & 0,798 & 0,913 \\
\hline $\mathrm{C7}$ & 1,03 & 1,58 & 1,113 & 1,163 \\
\hline $\mathrm{C} 8$ & 1,62 & 2,76 & 0,895 & 0,924 \\
\hline $\mathrm{C9}$ & 1,51 & 0,987 & 1,90 & 0,989 \\
\hline C10 & 1,52 & 1,028 & 2,06 & 1,067 \\
\hline C11 & 1,62 & 2,02 & 1,141 & 1,015 \\
\hline $\mathrm{C} 12$ & 2,10 & 2,73 & 1,060 & 0,922 \\
\hline $\mathrm{C} 13$ & 2,49 & 3,17 & 1,103 & 0,876 \\
\hline
\end{tabular}

Entre los alumnos del Plan Antiguo, correspondientes a la antigua titulación de Diplomatura en Magisterio, se percibe con más claridad la adquisición de competencias tales como "Elaborar diseños curriculares" $(2,49)$ y "Fomentar la educación en valores" $(2,10)$, seguidas a cierta distancia por "Fomentar el interés por la lectura" $(2,10)$ y "Utilizar las tecnologías de la información y la comunicación" $(1,62)$. Mientras que la percepción de las competencias menos adquiridas correspondería, por orden, a "Participar en la gestión del Centro" $(0,75)$, "Informar e implicar a las familias" $(1,03)$, "Gestionar la progresión de los aprendizajes" $(1,40)$ y "Trabajar en equipo con otros docentes" $(1,42)$.

Los alumnos del Plan Nuevo perciben más claramente la adquisición de competencias tales como "Elaborar diseños curriculares" $(3,17)$, "Utilizar las tecnologías de la información y la comunicación" $(2,76)$ y "Fomentar la educación en valores" $(2,73)$. Por otro lado, la percepción de las competencias menos adquiridas recaería, por orden, en "Afrontar los deberes y los dilemas éticos de la profesión" $(0,98)$, "Organizar la propia formación continua" $(1,02)$, "Participar en la gestión del Centro" $(1,29)$, seguido de "Informar e implicar a las familias" $(1,58)$.

Tabla 5- Resumen de la percepción de las competencias adquiridas por parte de los alumnos durante la formación inicial del profesorado. 


\begin{tabular}{||l||l|l|l||}
\hline & $\begin{array}{l}\text { Alumnos del } \\
\text { Plan antiguo }\end{array}$ & $\begin{array}{l}\text { Alumnos del } \\
\text { Plan nuevo }\end{array}$ & $\begin{array}{l}\text { Alumnos del Plan nuevo, } \\
\text { después del ejercido de la } \\
\text { profesión }\end{array}$ \\
\hline \hline Corcepción & & & $\mid \mathrm{C} 3$ \\
& $\mathrm{C} 13$ & $\mathrm{C} 13$ & $\mathrm{C} 2$ \\
$\mathrm{C} 11$ & $\mathrm{C} 8$ & $\mathrm{C} 12$ \\
\hline & $\mathrm{C} 8$ & $\mathrm{C} 2$ & $\mathrm{C} 10$ \\
& $\mathrm{C} 6$ & $\mathrm{C} 9$ & $\mathrm{C} 6$ \\
& $\mathrm{C} 7$ & $\mathrm{C} 10$ & $\mathrm{C} 8$ \\
& $\mathrm{C} 2$ & $\mathrm{C} 6$ & $\mathrm{C} 7$ \\
\hline
\end{tabular}

Los primeros resultados generales del análisis cualitativo entre los egresados de Grado que ejercen o han ejercido la profesión, nos muestran un cambio en la percepción de las competencias que más adquirieron durante su formación de Grado. Seis años después, continúan manteniendo la percepción de que la competencia "Fomentar la educación en valores" fue adquirida en la mayor parte de las asignaturas durante la carrera. Sin embargo, tras el ejercicio profesional, existe un cambio de percepción a favor de "Gestionar la progresión de los aprendizajes" y "Elaborar y poner en práctica estrategias de atención a la diversidad".

De las competencias que actualmente perciben que fueron menos desarrolladas durante su formación inicial todavía se mantienen dos: menos valoradas son "Organizar la propia formación continua" y "Participar en la gestión del centro". Destaca un cambio interesante en la percepción sobre la competencia "Utilizar las tecnologías de la información y la comunicación" que fue una de las consideradas como más adquiridas mientras eran alumnos de Grado y ahora, tras el ejercicio de la profesión, pasa a ser considerada una de las menos adquiridas.

A pesar de todo, consideran que las tres competencias que el profesorado debe continuar trabajando mucho más durante la Formación Inicial son las competencias que permitirían al futuro docente mejorar en "Elaborar y poner en práctica estrategias de atención a la diversidad", "Gestionar la progresión de los aprendizajes" y "Organizar y animar situaciones de aprendizaje".

\section{Discusión}

Los principales resultados obtenidos de la investigación cuantitativa y cualitativa a los alumnos, ya egresados, respecto a la adquisición de las competencias docentes durante su periodo formativo, nos llevan a concluir:

La nueva forma de trabajo del profesorado universitario dentro del aula que requiere el Plan Bolonia ha contribuido a un cierto cambio en la percepción de la adquisición de competencias entre los alumnos, ya egresados, del plan antiguo y los del plan nuevo. Aunque se mantiene en la universidad la tendencia a formar a los futuros docentes en aquellas competencias que facilitan el desempeño de sus tareas dentro del aula.

Las competencias adquiridas que en general han sido percibidas más claramente por los alumnos durante la Formación Inicial son aquellas que los futuros docentes pueden desarrollar dentro del aula (elaboración diseños curriculares, fomentar la educación en valores, etc.) quedando relegadas todas aquellas que les capacitarían mejor en la resolución de situaciones profesionales habituales para avanzar en las relaciones con 
otros miembros de la comunidad educativa, y que tendrían una mayor relevancia fuera del aula, como pueden ser trabajar en equipo con otros docentes, participar en la gestión de la escuela o la implicación de las familias en el centro.

El hecho de estar ejerciendo la profesión también influye en la percepción de las competencias que se adquirieron durante los estudios de Grado. Los ahora ya profesionales evalúan la percepción de las competencias que adquirieron desde un punto de vista más práctico, basado en situaciones y necesidades reales, aunque continúa persistiendo la demanda al profesorado, en mayor medida, de trabajar aún más las competencias necesarias para la realización de su propio trabajo dentro del aula.

En este sentido, el marco actual de la sociedad del conocimiento que anima a implementar cada vez más el uso de las NTIC dentro del aula, el profesor novel es consciente del papel más activo que tiene que ir asumiendo en la construcción del propio conocimiento. Esto se traduce en una mayor demanda en la aplicación concreta de las NTIC en la elaboración de unidades didácticas y la gestión del conocimiento y de los aprendizajes.

Es necesario continuar profundizando en este tipo de estudios con el fin de mejorar en la universidad la formación del alumnado basada en competencias. El término competencias requiere al entorno universitario una mayor adecuación entre lo que se estudia en la universidad y el ejercicio de la profesión, sin que ello menoscabe la independencia de la institución frente a las demandas neoliberales del mercado y en aras a lograr una transformación de la sociedad hacia un modelo más justo y equitativo para todos.

La identificación de las competencias a trabajar y adquirir por los futuros maestros, tarea no exenta de valores éticos que debiera realizarse desde el ámbito universitario, y el grado de adecuación entre las competencias que adquieren y las que después tendrán que desarrollar permite a la universidad asumir, en mayor y mejor medida, la responsabilidad social que adquiere con los futuros maestros durante su Formación Inicial y con la sociedad en general.

Para ello, también es necesario incorporar el punto de vista de otros actores clave en la Formación Inicial como los propios profesores universitarios o los tutores de prácticas, que participan también desde sus centros en la Formación Inicial, que permitirá analizar la coherencia entre el desarrollo, la adquisición y las necesidades reales en materia de competencias docentes.

\section{Referencias}

Martínez, M. (2016). La formación inicial de los maestros: una responsabilidad compartida. Bordón. Revista de Pedagogía, $68(2), 9-16$

Montero, L. (2001). La construcción del conocimiento profesional docente. Rosario: Homo Sapiens Ediciones.

Pavié, A. (2011). Formación docente: hacia una definición del concepto de competencia profesional docente. REIFOP, 14(1), $67-80$.
Perrenoud, P. (1999). Construir competencias desde la escuela. Santiago de Chile: Dolmen.

Perrenoud, P. (2004): Diez nuevas competencias para enseñar. Barcelona: Ed. GRAÓ.

Pimienta Prieto, J.H. (2012) Las competencias en la docencia universitaria. Preguntas frecuentes. México: Pearson.

Zabalza, M. A. (2012). Las competencias en la formación del profesorado: de la teoría a las propuestas prácticas. Tendencias Pedagógicas, 20, 5-32. 